\title{
Semiotische Merkmale in der Werbung von türkischen und deutschen alkoholischen Getränken
}

\author{
Türkiye ve Almanya'daki Alkollü İçki Reklamlarında Göstergebilimsel Özellikler
}

\section{Didem PIRCANLI* Tahir BALCI**}

Zusammenfassung: In diesem Beitrag werden semiotische Merkmale in der Werbung von türkischen und deutschen alkoholischen Getränken analysiert. Hierfür werden zuerst die Zeichentheorien von F. de Saussure, C. S. Peirce ve C. W. Morris dargestellt. Dann werden jeweils zwei Bierwerbungen, und zwar die Werbung für Veltis in Deutschland und die Werbung für Efes Pilsen in der Türkei kontrastiert. Anschliessend werden die Gemeinsamkeiten und Unterschiede festgestellt und die Ergebnisse kommentiert. Es hat sich herausgestellt, dass beide Biermarken das Blau hervorheben. Wir nehmen an, dass diese Farbe daher vorgezogen wurde, weil sie eher Gelassenheit, Ruhe, Friede und Sehnsucht assoziiert. In der Efes-Werbung wird durch das Blond indirekt auf blonde Frauen hingewiesen, was als ein soziokulturelles Motiv sexuellen Missbrauchs betrachtet werden kann. Das Blond in der Werbung von Veltis dagegen lässt eine solche geschlechtsspezifische Unterscheidung nicht erkennen.

Schlüsselwörter: Semiotik, Werbesprache, Alkoholwerbung, kontrastive Analyse

Özet: Bu çalışmada, Türkçe ve Almanca alkollü içki reklamlarının göstergebilimsel özellikleri irdelenmektedir. Bu amaçla önce F. de Saussure, C. S. Peirce ve C. W. Morris’in görüşleri ışığında göstergebilime ve gösterge türlerine genel bir bakıştan sonra Almaya'da tüketilen Veltins ile Türkiye'de tüketilen Efes Pilsen biralarının ikişer reklamı karşılaştırılmıştır. Bir sonraki aşamada reklamlar arasındaki göstergebilimsel benzerlikler ve farklıklar belirlenmeye, bulguların gerekçelendirilmesine çalışılmıştır. Sonuç olarak Veltins ve Efes Pilsen'in reklamlarında hâkim renk olarak maviyi tercih ettikleri görülmüsstür. Bu tercihin, mavi rengin sükûnet, huzur, barış, özlem gibi olumlu çağrışımlar yaratmasından kaynaklandığı fikrine varılmıştır. Efes birasının bir reklamında ise sarı rengin kullanılması yoluyla Türk erkeklerin genelde tercih ettikleri sarışın kadınla bağlantı kurulmuş, sosyokültürel bir motiften yararlanılmış, bir bakıma cinsellik sömürüsü yapılmıştır. Veltins’in kullandığı sarı rengin cinsiyet çağrıştırıcı özelliği yoktur.

Anahtar Sözcükler: Göstergebilim, reklam dili, içki reklamı, karşılaştırmalı çözümleme

\section{Einleitung}

In unserem alläglichen Leben gibt es einige Darstellungsformen, denen wir meistens keine Aufmerksamkeit schenken. Zu diesen Formen gehören semiotische Merkmale, die als semantische, syntaktische oder pragmatische Sprachelemente auf etwas außer sich selbst verweisen.

Durch das Fernsehen, die Zeitungen, das Radio, den Computer oder durch das Billboard befinden wir uns täglich inmitten eines Informationsdschungels. Es ist selbstverständlich, dass

\footnotetext{
*MA., Çukurova Üniversitesi, Sosyal Bilimler Enstitüsü, didempircanli@cu.edu.tr

** Prof. Dr., Çukurova Üniversitesi, Eğitim Fakültesi, Yabancı Diller Eğitimi Bölümü, tbalci@cu.edu.tr
} 
wir all diese Botschaften bzw. Darstellungen nicht bemerken oder bewerten können. Aus diesem Grund gibt es einen heimlichen Krieg, diese Botschaften im Gehirn zu speichern. An diesem Punkt tritt die Relevanz der Zeichen hervor. All diese Phänomene sieht man in der Werbesprache. Daher werden in diesem Beitrag die semiotischen Merkmale von türkischen und deutschen alkoholischen Getränken kontrastiv analysiert.

\section{Semiotik: Zeichentypen}

Die Semiotik wird kurz als Wissenschaft von Zeichen definiert. Sie beschäftigt sich mit allen Prozessen des Informationsaustauschs, die an der Entstehung der Zeichen beteiligt sind. Wenn wir verstehen wollen, was Semiotik ist, sollten wir zuerst wissen, was Zeichen ist.

F. de Saussure hat die Sprache als Zeichensystem angesehen (Hartmann, Pütz, \& Schofe, 1978, 21). Ein Zeichen ist nach ihm eine arbiträre konventionelle Einheit von Lautbild und Vorstellung. In der Alltagssprache wird das (Sprach-) Zeichen im Allgemeinen als etwas verstanden, das eine Bedeutung hat und für etwas anderes steht (vgl. Lewandowski, 1994). Wir können sagen, dass jede Erscheinung, die etwas anderes ausdrückt und nicht nur als ein Phänomen aufzufassen ist, als ein Zeichen betrachtet werden kann. Insofern gelten die Wörter, die Symbole, die Attribute usw. als Zeichen.

Alle Sprachen, die gesellschaftlich sind und zwischenmenschliche Kommunikation verschaffen, bestehen aus den Beziehungen zwischen den Zeichen-Zeichen, Zeichen-Menschen und Zeichen(-körper)-Zeicheninhalt.

\section{Abbild 1: Sprachzeichen}

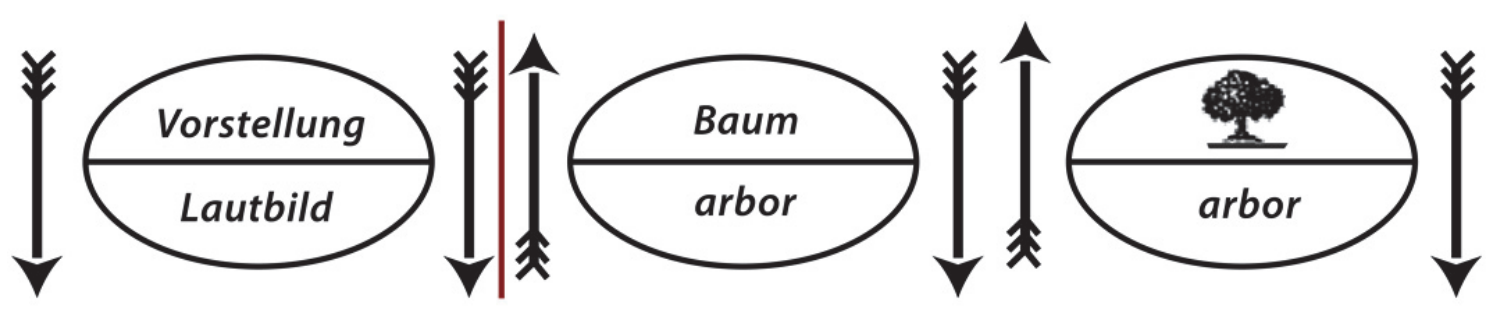

"Diese beiden Bestandteile sind eng miteinander verbunden und entsprechen einander. Ob wir nun den Sinn des lat. Wortes suchen oder das Wort, womit das Lateinische die Vorstellung" Baum "bezeichnet, so ist klar, dass uns nur die in dieser Sprache geltenden Zuordnungen als angemessen erscheinen, und wir schließen jede beliebige andere Zuordnung aus, auf die man sonst noch verfallen könnte" (Hartmann, 1978, 46).

Neben de Saussure ist Charles Sanders Peirce einer der Begründer der Semiotik. Der Begriff Zeichen wird von Peirce als eine triadische bzw. dreistellige Relation zwischen einem Repräsentanten, einem Interpretanten und einem Objekt eingeführt. Ein Repräsentamen wird als ein Zeichenträger, ein Interpretant wird als ein Gedanke definiert (Abbild 2; www.semiot.wordpress.com).

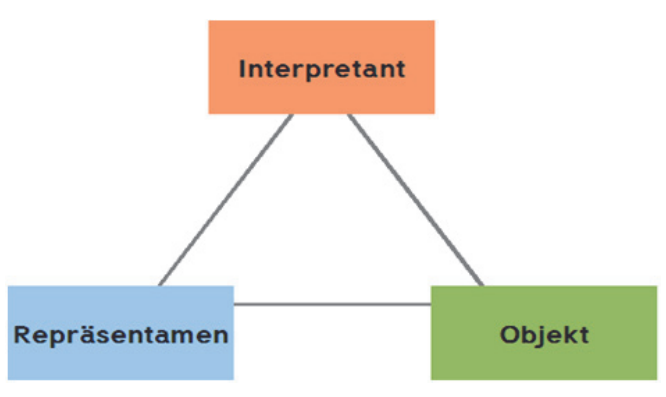

\section{Abbild 2}

"Ein Zeichen oder Repräsentamen ist alles, was in einer solchen Beziehung zu einem Zweiten steht, das sein Objekt genannt wird, dass es fähig ist, ein Drittes, das sein Interpretant genannt wird, dahin gehend zu bestimmen, in derselben triadischen Relation zu jener Relation auf das Objekt zu stehen, in der es selber steht. Dies bedeutet, dass der Interpretant selbst ein Zeichen 
ist, das ein Zeichen desselben Objekts bestimmt und so fort ohne Ende" (Peirce, 1993, 64).

Charles W. Morris ist ein anderer wichtiger Name der Semiotik. Er entwickelte seine Zeichentheorie unter behavioristischen Aspekten. Bei seiner Zeichentheorie handelt es sich um die Dreiteilung der Zeichen in Syntaktik, Semantik und Pragmatik. Das Verhalten wird mit Hilfe dieser drei Richtungen untersucht (vgl. Gross, 1990, 31). Von dieser Betrachtungsweise ausgehend kann man sagen, dass die Alphabete, die Verkehrszeichen, die Werbeplakate, die Bilder, die Musik, das Verhalten, die Gestik, die Mimik, die Symbole und deren Einheiten Zeichen sind.

Die Menschen sind soziale Wesen. Die Kommunikation ist das wichtigste Phänomen, das sie verbindet. Durch sie wird das Zeichen erzeugt. Aber man soll den Anwendungsort von Zeichen kennen, um sie angemessen benutzen zu können.

\subsection{Symbol, Icon, Indices}

Die Zeichen werden von Ch. S. Peirce in drei Klassen unterteilt. Diese sind Symbol, Icon und Indices. Die Klassifizierung von Zeichen ist ein wichtiger Beitrag von Peirce zur Semiotik. Symbole "haben eine rein bezeichnende Funktion, einen allgemeinen Sinn ohne Ähnlichkeit mit dem Objekt." (Gross, 1990, 30). Ikone haben dagegen "eine abbildende Funktion und deshalb eine gewisse Ähnlichkeit mit ihren Objekten.” (ebd). Indices "haben eine anzeigende Funktion, sie verweisen auf etwas Individuelles" (ebd.).

Abbild 3.

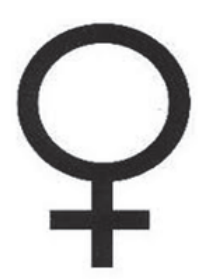

'weiblich'

(Symbol)
Abbild 4.

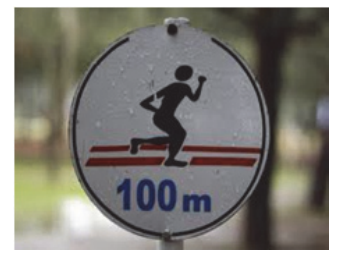

'sprint'

(Icon)
Abbild 5.

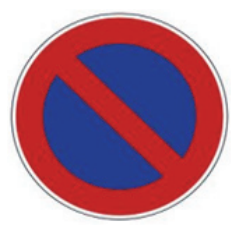

'parkverbot' (Indices)

\subsection{Sprachliche (verbale) - nichtsprachliche (nonverbale) Zeichen}

Neben Symbolen, Iconen und Indices gibt es noch sprachliche und nicht sprachliche Zeichen. Alles, was wir beim Kommunizieren verwenden, ist sprachliches Zeichen. Nichtsprachliche Zeichen sind im weiteren Sinne beispielsweise Gestik, Mimik und Körperhaltung, die sich in einer sprachlichen Äußerung manifestieren und unabhängig von der Sprache existieren.

\section{Was ist Werbung?}

Nach Bechstein $(1987,11)$ ist Werbung der planmäßige Einsatz von Personen, Mitteln und Techniken zur gezielten Beeinflussung menschlichen Verhaltens, zur Weckung von Bedürfnissen oder Verbreitung ideeller Güter. Ziel der Werbung ist die Erfüllung eines materiellen oder immateriellen Zwecks. Sie ist eine Kommunikationsform, bei der zumeist mehrere Zeichensysteme gleichzeitig Verwendung finden. Sie ist also eine Form symbolischer Kommunikation, weil sie Produkte darstellt, die "nicht physisch greifbar" sind (Schweiger, \& Schrattenecker, 1992, 8). Daher sollen die Werbemacher die semiotischen und kulturellen Gegebenheiten, die für die Vermarktung eines Produktes relevant sind, auf jeden Fall berücksichtigen.

Der Werbungprozess verweist vor allem darauf, dass es auf Teilen des Senders zu einem gemeinten Einsatz von Zeichenträgern zur Übertragung eines Zeicheninhaltes an einen Empfänger kommt. Die Nachrichten sind voneinander unterschiedlich. Eine von beiden wird vom Sender 
unter Benutzung eines Codes enkodiert. Eine andere wird vom Empfänger interpretiert. Also die eine ist enkodierte Nachricht, die andere ist dekodierte Nachricht (vgl. Moshbach, 2000, 38). Um den Dekodierungsprozess von Werbewirkung zu leiten und Fehlerinterpretationen zu vermeiden, werden die Werbedarstellungen anspruchsvoll ausgewählt. Ihr äußeres Bild muss sehr genau auf gewünschtem Identifikationsmodell passen. Die Werbegestalten fungieren als "bedeutungserzeugendes System" (vgl. Fischer-Lichte, 1983, 64).

Neben dem kommunikativen Aspekt muss die Werbung auch den kulturellen Hintergrund beachten, weil dieser zum großen Teil die Rezeption mitbestimmt.

Die Werbung versucht, das Verhalten von Konsumenten mittels raffinierter Kommunikationsmittel zu beeinflussen. In den Werbungen werden die Kenntnisse über das Leben, die Gedanken und die Gefühle von Menschen übermittelt.

Einer Werbung geht meistens eine Marktuntersuchung voraus, dessen Ziel die Bestimmung einer Zielgruppe ist. Die Eigenschaften, die Voraussetzungen und die Einkaufgründe der Zielgruppen werden festgelegt. "Die hauptsächlichen Ziele von Werbung sind, dass ein Angebot von den Zielgruppen bemerkt und beachtet wird, dass die Zielgruppen das Angebot positiv beurteilen und dass sie es in Anspruch nehmen" (Müller, 1999, 142).

Die Zielgruppen bestehen aus Personen, die vom Werbemacher am Ende der Werbung beeinflusst werden sollen. Die Kaufhandlung soll mehr und mehr durch Verbreitung subjektiver Inhalte, die mit dem Produkt verbunden sind, ausgelöst werden. Die Personen eignen sich in dieser Linie die neue Lebensweise, die neuen Werte und die neuen Produkte an: "Werbung verfolgt das Ziel, den Empfänger einer Werbebotschaft zu Konsumhandlungen zu bewegen" (Nöth, 1985, 449). Um diesen Zweck zu erreichen, werden die Gestaltungsmittel, die für die Werbeverwendung bereit sind, im Rahmen der Analyse noch näher betrachtet. Die Reihe der einzelnen Wirkungsschritte von Werbung wird in der viel zitierten AIDA-Formel (vgl. http://www.wirtschaftslexikon24.net/d/aida-formel/aida-formel.htm) klar gemacht: Zunächst soll die Aufmerksamkeit des Konsumenten erregt werden, damit er sich für das Produkt interessiert. Dann soll ein Traum, ein Wunsch, ein Bedürfnis provoziert werden. Zum Schluss sollen diese zur Kaufhandlung führen (Aktion).

Die Werbe-Kommunikation setzt also voraus, dass zuerst ein Produkt auf dem Markt bekannt gemacht wird. Dadurch werden bestimmte Informationen über das Produkt vermittelt. Damit werden eine Aufmerksamkeit und ein Interesse erregt. Dann wird versucht, ein emotionales Image aufzubauen. Damit wird ein Traum erzeugt. Zuletzt wird ein Angebot mit komplexen informativ-emotionalen Beurteilungen verbunden. Es führt den Konsumenten zur Kaufhandlung.

Also Werbung versucht, Aufmerksamkeit zu erlangen, indem sie sich im Gedächtnis der potentiellen Käufer einen Platz eröffnet und sie vom Nutzen und der Qualität des Produktes überzeugt. Den Kern der Werbebotschaft bildet der Appell zum Kauf.

\section{Werbung von türkischen und deutschen alkoholischen Getränken}

\subsection{Die Analyse der deutschen Werbung von "Veltins"}

Man sieht im Wasser einige Bierflaschen. Der Werbemacher muss für diese Getränkewerbung die blaue Farbe deswegen favorisiert haben, weil das Blau etwas Positives symbolisiert: Kühle, Frische, Ferne, Unendlichkeit, unbegrenzte Freiheit, Sehnsucht und Ruhe. Das Bier wird kühl getrunken. Das ist ein Appell, den wir nicht direkt wahrnehmen können. "Das ist ein kühles Bier. Kauf es!” Außerdem sind manche Flaschendeckel auf. Das bedeutet, dass das Bier trinkbereit ist. In dieser Hinsicht ist Veltins verlockend. 
Das bevorzugte Schlagwort ist kurz und einfach. Das Verb ,feiern' wird mit dem Graphem >V< geschrieben. Das ist ein kleines, aber intelligentes Detail, weil das große $>\mathrm{V}<$ auch die erste Schreibeinheit von Veltins ist. Wenn man also unaufhörlich feiern will, macht man es mit Veltins. Alle diese Bedeutungen sind konnotativ.

Die Flaschen können in dieser Werbung auch als Icone angenommen werden. Es gibt Übereinstimmung zwischen der Inhalts- und Ausdrucksseite. Aber der Slogan ist ein Symbol. Wenn wir eine Verbindung zwischen dem Produkt und dem Slogan bilden möchten, müssen wir zuerst die deutsche Sprache kennen. Sonst wäre die Werbung sinnlos.

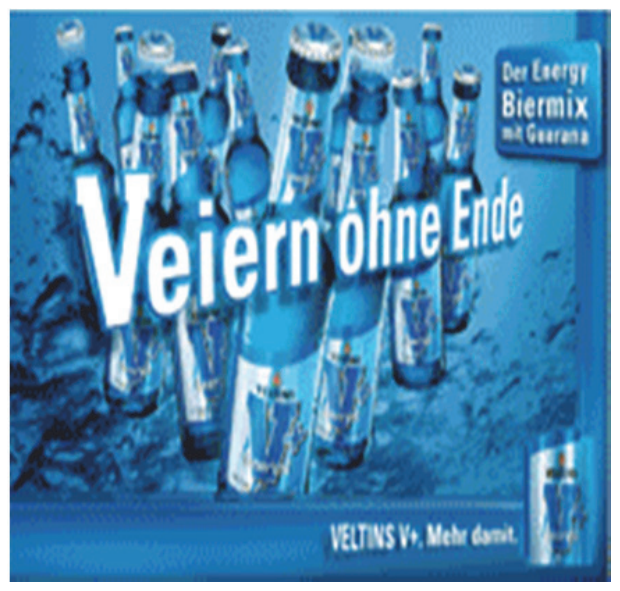

Abbild. 6 (www.getraenke-huesemann.de)

\subsection{Die Analyse der türkischen Werbung von "Efes Pilsen"}

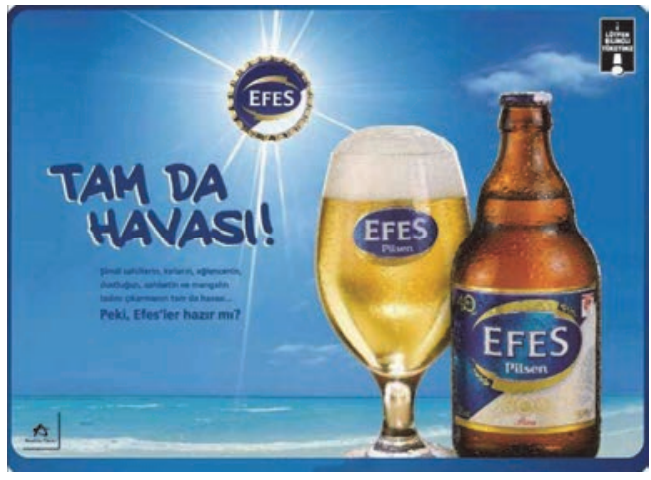

Abb. 7 (www.didikdidik.net)

Man sieht hier das Meer, den Himmel, eine Flasche und ein Glas Bier. Die Sonne wird als ein Bierflaschendeckel dargestellt. Genau so wie in der deutschen Bierwerbung dominiert auch hier die blaue Farbe. Wir haben schon gesagt, dass das Blau etwas Positives symbolisiert wie Kühle, Frische, Ferne, Unendlichkeit, unbegrenzte Freiheit etc. Aber hier gibt es noch zwei Erscheinungen, die diese Bedeutungen hervorheben: Das Meer und der Himmel. Diese zwei Begriffe symbolisieren zudem den Sommer. Die Werbung ist sowieso besonders für Sommermonate vorgesehen. Wir können es sowohl am Bild als auch am Slogan (Tam da Havası) erkennen: Es ist genau die Zeit, die Küste, die Freundlichkeit, die Unterhaltung und den Grill zu genießen (mit kleinen Buchstaben). Und sind auch die Efes da? Also hier gibt es einen Appell: Wenn es die Sommerzeit ist, wenn es warm ist, wenn man sich abkühlen möchte, wen man feiern möchte, dann muss man Efes konsumieren.

Die Gleichsetzung von Sonne und Bierflachendeckel ist eine sehr kreative Idee. Demnach kann der Konsument das Efes-Bier als die Quelle von Freude, Genuss und Gesundheit deuten.

Die Flasche, das Meer und der Himmel können in dieser Werbung als Icone angenommen werden. Es gibt Übereinstimmung zwischen der Inhalts- und Ausdrucksseite. Man kann ja verstehen, dass es eine Bier-Werbung ist. Aber der Slogan ist ein Symbol. Wenn wir eine Verbindung zwischen dem Produkt und dem Slogan bilden möchten, müssen wir zuerst die türkische Sprache kennen.

Der Unterschied zwischen der türkischen und der deutschen Werbung ist, dass man die türkische Sprache nicht beherrschen muss, um den Appell zu empfangen. Hier ist das Bild im Vordergrund, nicht der Slogan. Natürlich gibt es eine Verbindung

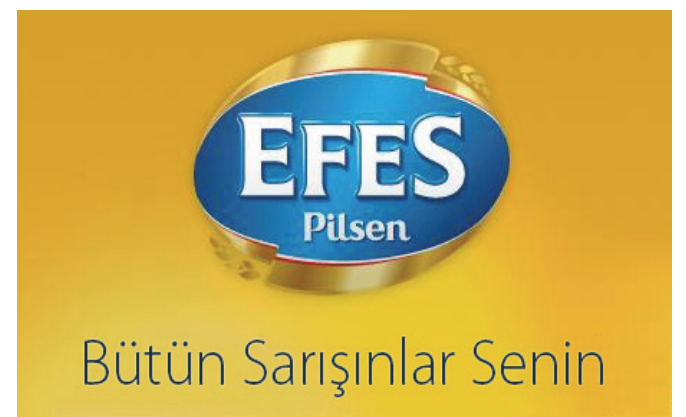

Abbild. 8 (www.yeni1blog.com) 
zwischen dem Slogan und dem Bild (der Bierflachendeckel), aber man kann eine Verbindung zwischen dem Inhalt des Slogans und dem Bild durch das Sehen bilden. Wir können sagen, dass die türkische Werbung mehr Menschen ansprechen und erreichen kann.

In der obigen Werbung sehen wir ein Emblem, das auf eine gelbe Oberfläche gesetzt ist; unter diesem Emblem steht der Slogan "Bütün Sarışınlar Senin”. Hier handelt es sich um eine einfache unkomplizierte Werbung ohne ansehnliche bemerkenswerte Bilder bzw. Figuren.

Hier wird die traditionelle blaue Farbe (der blaue Deckel) mit überwiegendem Goldgelb kombiniert, durch das auf blonde Frauen angespielt wird. Der Slogan "Bütün Sarışınlar Senin" vervollständigt diese Anspielung. Mit dem Slogan wird ein Zusammenhang zwischen Bier und blonden Frauen hergestellt.

Dieser Slogan nutzt den Sinn türkischer Konsumenten (Männer) für das Gegengeschlecht und legt den Unterschied zwischen dem Schönheitsverständnis von türkischen und deutschen Männern nahe. Man kann vermuten, dass der Werbemacher davon ausgeht, dass eine blonde Fraumeistens als ein schönes Frauenporträt für einen türkischen Mann gilt. Der Werbemacher sagt dem Konsumenten: Wenn eine blonde Frau eine schöne Frau ist und deinem ästhetischen Sinn entspricht, dann entspricht auch das Efes-Goldier deinem Geschmack. Man will hier den Geschmackssinn durch einen Geschlechtssinn erregen.

Der Slogan "Bütün Sarışınlar Senin” ist strukturell ambig. Dadurch kann er auf zweierlei Weise verstanden werden: a) Alle Blondinen gehören dir; b) Alles Efes-Goldbier gehört dir. Das ist kein Zufall; gerade hier liegt die Kreativität und die Effektivität dieser Werbung.

Wenn wir in Anlehnung an Peirces Zeichenklassen sprechen: Das Emblem "Efes" ist ein Symbol, weil es keine Ähnlichkeit zwischen Form und Inhalt gibt. Das Emblem ist ein Zeichen im engeren Sinne. Man muss die Marke kennen, um verstehen zu können, dass es sich um Bierwerbung handelt.

Der Slogan ist Symbol mit Indices, weil es keine Ähnlichkeit zwischen Form und Inhalt gibt; aber es verweist auf etwas. Der Werbemacher möchte einen Zusammenhang zwischen dem Produkt und dem Slogan bilden.

\section{Schlussbemerkung}

"Veltins" hat ja zahlreiche Werbungen, in denen auch das Blond als Haar- oder Bierfarbe vorkommt. Mit dem Blondgelb verweist man aber nicht auf Geschlecht oder Sexualität.

Wenn wir die Werbungen von Veltins und Efes vergleichen: Beide bevorzugen das Blau als Grundfarbe. Nur in der zweiten Efes-Werbung wird das Goldgelb geschlechtsspezifisch als Sinnbild für blonde Frauen verwendet. In der Werbung von Veltins wird aber durch Goldgelb die lange Brautradition hervorgehoben, die durch Jahresangabe "seit 1824" bestätigt wird und fast alle Biermarken in den Vordergrund stellen. Der Text unter dem Bild betont die Frische und das Anderssein von Veltins.

In der ersten Werbung von Veltins (Abb. 6) werden die Bierflaschen als Ikon benutzt. Auch in der ersten EfesWerbung (Abb. 7) werden Ikone verwendet, in der zweiten dagegen nicht.

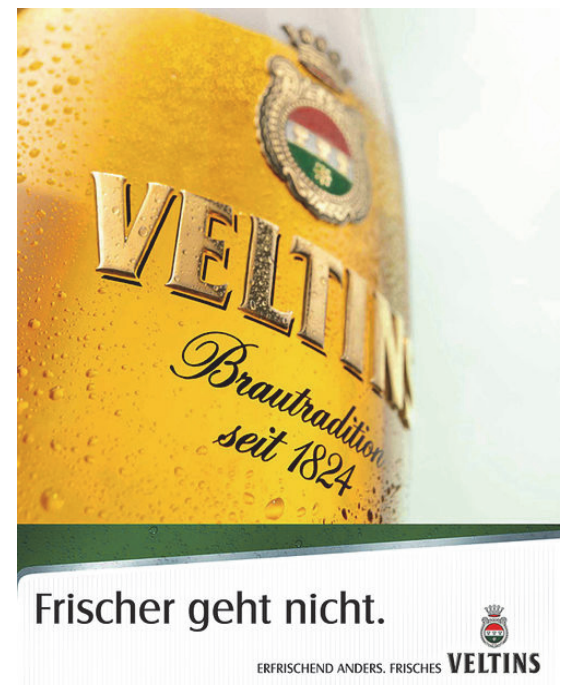

Abbild. 9

(http://www.veltins.de/\#app=113e\&7c ad-siteId=veltinsSpecial) 
In beiden Werbungen (Abb. 6 und 7) stehen die Slogans im Mittelpunkt. Die Slogans sind kreativ und wirksam. Mit dem Slogan der ersten Werbung von Veltins (Abb. 6) möchte man eine Verbindung zwischen Freude und Bier herstellen, ungefähr so wie bei der ersten EfesWerbung. Die mit der zweiten Efes-Werbung (Abb. 8) beabsichtigte Parallelität zwischen schönen blonden und schönem Efes-Goldbier deutet auf einen kulturellen Unterschied zwischen Deutschen und Türken und stellt die Frau herabschätzend als eine Konsum-Sache dar.

In allen Werbungen will der Werbemacher dem Konsumenten die Vollkommenheit des Produktes einprägen und ihn dazu bewegen, das Produkt zu kaufen. Das wird durch visuelle, verbale oder schriftliche Mittel versucht. Diese Mittel spiegeln zugleich die sozio-kulturellen Eigenschaften der Konsumenten wider. Anhand dieser Untersuchung wurde deutlich gezeigt, dass besonders visuelle semiotische Merkmale die grundlegenden Bausteine jedweder Werbung sind.

\section{ABKÜRZUNGEN und QUELLEN}

Bechstein, G. (1987). Werbliche Kommunikation. Grund Informationen zur semiotischen Analyse von Werbekommunikation. Bochum: Studienverlag Dr. N. Brockmeyer.

Fischer-Lichte, E. (1983). Semiotik des Theaters Bd.1, Das System der theaterischen Zeichen. Tübingen: Narr Verlag.

Gross, H. (1990). Einführung in die germanistische Linguistik. München: Indicium Verlag.

Hartmann, W., Pütz, H.-H., \& Schofe, P. (1978). Sprachwissenschaft für den Unterricht. Schwann, Düsseldorf: Pädagogischer Verlag.

Lewandowski, T. (1994). Linguistisches Wörterbuch. 6. Aufl. Quelle, \& Meyer: Heidelberg.

Mosbach, D. (1998). Bildermenschen-Menschenbilder. Exotische Menschen als Zeichen in der neueren deutschen Printwerbung. Berlin: Berlin-Verlag.

Müller, W. G. (1999). Semiotik der Werbung. Deutschland: Staufenburg.

Nöth, W. (1985). Handbuch der Semiotik. Stuttgart: J. B. Metzlersche Verlagsbuchhandlung und Carl Ernst Poeschel Verlag.

Peirce, C. S. (1993). Phänomen und Logik der Zeichen. Frankfurt a.M: Suhrkamp.

Schweiger, G., \& Schrattenecker, G. (1992). Werbung. 3.Verlag, Stuttgart: Gustav Fischer Verlag.

\section{Internetquellen:}

http://www.didikdidik.net/?p=1248 (Zugriff am 11.06.2012).

http://semiot.wordpress.com/2008/01/07/peirce-semiosis-ein-prozess-aus-zeichen-objekt-undinterpretant/ (Zugriff am 11.06.2012).

http://www.getraenke-huesemann.de/cms/index.php?page=impressum (Zugriff am 11.06.2012).

http://www.wirtschaftslexikon24.net/d/aida-formel/aida-formel.htm (Zugriff am 07.11.2012).

http://www.veltins.de/\#app=113e\&7cad-siteId=veltinsSpecial (Zugriff am 07.11.2012). 
